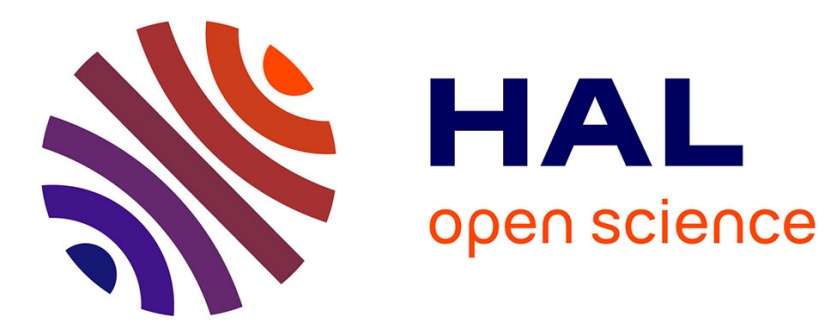

\title{
Kupferchalkogenid-Clusterverbindungen mit Brom-funktionalisierter Ligandenhülle
}

Robert Langer, Linda Wünsche, Dieter Fenske, Olaf Fuhr

\section{To cite this version:}

Robert Langer, Linda Wünsche, Dieter Fenske, Olaf Fuhr. Kupferchalkogenid-Clusterverbindungen mit Brom-funktionalisierter Ligandenhülle. Journal of Inorganic and General Chemistry / Zeitschrift für anorganische und allgemeine Chemie, 2009, 10.1002/zaac.200900233 . hal-00512211

\section{HAL Id: hal-00512211 \\ https://hal.science/hal-00512211}

Submitted on 28 Aug 2010

HAL is a multi-disciplinary open access archive for the deposit and dissemination of scientific research documents, whether they are published or not. The documents may come from teaching and research institutions in France or abroad, or from public or private research centers.
L'archive ouverte pluridisciplinaire HAL, est destinée au dépôt et à la diffusion de documents scientifiques de niveau recherche, publiés ou non, émanant des établissements d'enseignement et de recherche français ou étrangers, des laboratoires publics ou privés. 


\section{Zeitschrift für Anorganische und}

Allgemeine Chemie

\section{Kupferchalkogenid-Clusterverbindungen mit Brom- funktionalisierter Ligandenhülle}

\begin{tabular}{|c|c|}
\hline Journal: & Zeitschrift für Anorganische und Allgemeine Chemie \\
\hline Manuscript ID: & zaac. 200900233.R1 \\
\hline Wiley - Manuscript type: & Article \\
\hline $\begin{array}{r}\text { Date Submitted by the } \\
\text { Author: }\end{array}$ & 25-Jun-2009 \\
\hline Complete List of Authors: & $\begin{array}{l}\text { Langer, Robert; Universität Karlsruhe, Institut für Anorganische } \\
\text { Chemie } \\
\text { Wünsche, Linda; Universität Karlsruhe, Institut für Anorganische } \\
\text { Chemie } \\
\text { Fenske, Dieter; Universität Karlsruhe, Institut für Anorganische } \\
\text { Chemie } \\
\text { Fuhr, Olaf; Forschungszentrum Karlsruhe, Institut für } \\
\text { Nanotechnologie }\end{array}$ \\
\hline Keywords: & Copper, Selenium, Sulphur, Cluster compounds, Crystal structure \\
\hline
\end{tabular}

\section{(5) ScholaroNE \\ Manuscript Central}




\title{
ARTICLE
}

DOI: 10.1002/zaac.200((will be filled in by the editorial staff))

\section{Kupferchalkogenid-Clusterverbindungen mit Brom-funktionalisierter}

\section{Ligandenhülle}

\section{Copper Chalcogenide Cluster Compounds with Bromo-functionalized Ligand Shell}

Robert Langer $^{\mathrm{a}}$, Linda Wünsche ${ }^{\mathrm{a}}$, Dieter Fenske ${ }^{\mathrm{a}, \mathrm{b}}$ und Olaf Fuhr ${ }^{\mathrm{a}, \mathrm{b}, *}$

Karlsruhe, ${ }^{a}$ Institut für Anorganische Chemie sowie DFG-Centrum für funktionelle Nanostrukturen (CFN) der Universität (TH) und ${ }^{\mathrm{b}}$ Institut für Nanotechnologie (INT) am Forschungszentrum Karlsruhe

Bei der Redaktion eingegangen am

\begin{abstract}
Five new copper chalcogenide cluster molecules, $\quad\left[\mathrm{Cu}_{4}\left(\mathrm{~S}-\mathrm{C}_{6} \mathrm{H}_{4}-\mathrm{Br}\right)_{4}\left(\mathrm{PPh}_{3}\right)_{4}\right] \quad(\mathbf{1}), \quad\left[\mathrm{Cu}_{22} \mathrm{Se}_{6}(\mathrm{~S}-\right.$ $\left.\left.\mathrm{C}_{6} \mathrm{H}_{4}-\mathrm{Br}\right)_{10}\left(\mathrm{PPh}_{3}\right)_{8}\right] \quad$ (2), $\quad\left[\mathrm{Cu}_{28} \mathrm{Se}_{6}\left(\mathrm{~S}-\mathrm{C}_{6} \mathrm{H}_{4}-\mathrm{Br}\right)_{16}\left(\mathrm{PPh}_{3}\right)_{8}\right]$ (3), $\quad\left[\mathrm{Cu}_{47} \mathrm{Se}_{10}\left(\mathrm{~S}_{-} \mathrm{C}_{6} \mathrm{H}_{4}-\mathrm{Br}\right)_{21}(\mathrm{OAc})_{6}\left(\mathrm{PPh}_{3}\right)_{8}\right] \quad$ (4) and $\left[\mathrm{Cu}_{8}\left(\mathrm{~S}_{-} \mathrm{C}_{6} \mathrm{H}_{4}-\mathrm{Br}\right)_{6}\left(\mathrm{~S}_{2} \mathrm{C}-\mathrm{NMe}_{2}\right)_{2}\left(\mathrm{PPh}_{3}\right)_{4}\right]$ (5) have been synthesized and characterized by single crystal X-ray structure analysis. 1-4 were prepared from the reaction of $\mathrm{CuOAc}, \mathrm{Br}_{-} \mathrm{C}_{6} \mathrm{H}_{4}-\mathrm{SSiMe}_{3}$ and $\mathrm{Se}\left(\mathrm{SiMe}_{3}\right)_{2}$ in the presence of $\mathrm{PPh}_{3}$. In a further reaction of $\mathbf{1}$ with ${ }^{\mathrm{i}} \mathrm{PrMgCl}$ and $\left(\mathrm{Me}_{2} \mathrm{~N}-\mathrm{CS}_{2}\right)_{2}$ cluster 5 was crystallized.
\end{abstract}

Keywords: Copper; Selenium; Sulphur; Cluster compounds; Crystal structure 
* Dr. O. Fuhr

Institut für Nanotechnologie (INT), Forschungszentrum Karlsruhe

Postfach 3640

D-76021 Karlsruhe (Germany)

Tel.: (+49) 07243/82-6441

E-mail: olaf.fuhr@int.fzk.de 


\section{Einleitung}

In der Literatur gibt es inzwischen eine Vielzahl von Publikationen über die Darstellung und Funktionalisierung von Nanopartikeln.[1] Beispielsweise finden sich darunter Arbeiten, in denen über eine Funktionalisierung von Metallnanopartikeln oder die Dimerisierung von Goldnanopartikeln mittels verbrückender Moleküle berichtet wird.[2] Obwohl es inzwischen schon viele Beispiele für die Synthese großer Cluster-Moleküle gibt,[3-8] wurde der Einfluss von funktionellen Gruppen in der Ligandenhülle solcher Cluster bislang wenig untersucht. Die meisten bisher publizierten Arbeiten beschäftigen sich mit Ligandenaustauschreaktionen.[9]

In unserer Arbeitsgruppe interessieren wir uns seit geraumer Zeit für die Synthese von Münzmetallchalkogenid-Clustern. Dazu setzen wir Salze des Typs MX (M = Cu, Ag, Au; X = OAc, Cl, OTf oder $\left.\mathrm{O}_{2} \mathrm{CPh}\right)$ mit einfach und zweifach silylierten Chalkogenverbindungen in Gegenwart tertiärer Phosphane um. Neben der Funktionalisierung der daran beteiligten Phosphane [10] bemühen wir uns um die Funktionalisierung der eingesetzten Phenylthiolate. So führte die Variation der funktionellen Gruppen zu veränderten Löslichkeitseigenschaften oder zur Bildung neuartiger MünzmetallchalkogenidCluster.[11,12] Auch die optischen Eigenschaften der synthetisierten Cluster ändern sich in Abhängigkeit von den funktionellen Gruppen.[13]

In der vorliegenden Arbeit berichten wir über die Synthese einer Reihe von Brom-funktionalisierten KupferchalkogenidClustern und deren Reaktivität bzw. Stabilität gegenüber Reagenzien, von welchen bekannt ist, dass sie mit Bromsubstituierten Aromaten reagieren.

\section{Ergebnisse und Diskussion}

Die Reaktion von Kupfer(I)-acetat mit $p-\mathrm{Br}-\mathrm{C}_{6} \mathrm{H}_{4}-\mathrm{SSiMe}_{3}$ und $\mathrm{Se}\left(\mathrm{SiMe}_{3}\right)_{2}$ in Gegenwart tertiärer Phosphane führt zur Bildung verschiedener Brom-funktionalisierter Kupferchalkogenid-Cluster. Die Bildung des jeweiligen Clusters ist dabei stark von der Stöchiometrie der eingesetzten Edukte abhängig. Führt man die beschriebenen Reaktionen von CuOAc und $\mathrm{PPh}_{3}$ mit $p$-Br- $\mathrm{C}_{6} \mathrm{H}_{4}-\mathrm{SSiMe}_{3}$ (im Verhältnis 1:1:1) ohne Zugabe von $\mathrm{Se}(\mathrm{SiMe})_{2}$ durch, so erhält man Verbindung 1 als Reaktionsprodukt. Wählt man hingegen andere Bedingungen und Mengenverhältnisse und verwendet zusätzlich noch $\mathrm{Se}\left(\mathrm{SiMe}_{3}\right)_{2}$ als Chalkogenidquelle, so erhält man verschiedene Kupferchalkogenid-Cluster mit Selenidkern und einer Thiolathülle. Schema 1 fasst die Reaktionen, die zu den Verbindungen 1-4 führen, zusammen.

Da mit zunehmender Größe der Cluster, und damit zunehmender Anzahl an funktionellen Gruppen, zu befürchten ist, dass die zu testenden Reaktionen wesentlich uneinheitlicher verlaufen und damit die Charakterisierung der Reaktionsprodukte erschwert wird, wurden an Verbindung 1 aufgrund der geringsten Anzahl an funktionellen Gruppen pro Molekül zunächst einige Untersuchungen zum Reaktionsverhalten gegenüber organometallischen Reagenzien durchgeführt, wobei eventuell 

nachgewiesen werden sollten. Bei der Umsetzung von Verbindung 1 (gelöst in DME) mit verschiedenen Mengen ${ }^{n}$ BuLi bei $-78^{\circ} \mathrm{C}$ konnte nach dem Erwärmen auf Raumtemperatur nur die Zersetzung von $\mathbf{1}$ beobachtet werden. So ergab das Abfangen dieser Reaktionen mit $\mathrm{D}_{2} \mathrm{O}$ bei unterschiedlich Temperaturen keine Veränderungen im ${ }^{1} \mathrm{H}-$ und ${ }^{13} \mathrm{C}\left\{{ }^{1} \mathrm{H}\right\}$-NMRSpektrum. Im Falle eines Brom-Lithium-Austausches würde man nach Zugabe von $\mathrm{D}_{2} \mathrm{O}$ im ${ }^{1} \mathrm{H}-\mathrm{NMR}$-Spektrum einen veränderten Signalsatz für die aromatischen Protonen, sowie eine zusätzliche Kopplung zum eingeführten Deuterium erwarten. Im ${ }^{13} \mathrm{C}\left\{{ }^{1} \mathrm{H}\right\}$-NMR-Spektrum müsste in diesem Fall eine Aufspaltung des para-ständigen Kohlenstoffatoms zum Triplett beobachtet werden. Ähnliche Beobachtungen wurden auch bei den Umsetzungen von 1 mit ${ }^{i} \mathrm{PrMgCl}$ gemacht. Hier zeigten die NMR-Spektren ebenfalls keine Veränderung nach der Zugabe von $\mathrm{D}_{2} \mathrm{O}$ bei verschiedenen Temperaturen. Verwendet man statt $\mathrm{D}_{2} \mathrm{O} \quad \mathrm{Me}_{3} \mathrm{SiCl}$ als Elektrophil zum Abfangen einer eventuell gebildeten organometallischen Zwischenstufe, so erhält man $\left[\mathrm{CuCl}\left(\mathrm{PPh}_{3}\right)\right]_{4}$ als Reaktionsprodukt. Bei der Reaktion von 1 mit ${ }^{i} \mathrm{PrMgCl}$ und anschließender Zugabe von $\left(\mathrm{Me}_{2} \mathrm{~N}-\mathrm{CS}_{2}\right)_{2}$, konnte hingegen eine Verbindung der Zusammensetzung $\left[\mathrm{Cu}_{8}\left(p-\mathrm{S}-\mathrm{C}_{6} \mathrm{H}_{4}-\mathrm{Br}_{6}\left(\mathrm{~S}_{2} \mathrm{CNMe}_{2}\right)_{2}\left(\mathrm{PPh}_{3}\right)_{4}\right]\right.$ (5) isoliert werden. Diese bildet sich aus zwei Molekülen von Verbindung 1, wobei zweier Thiolatoliganden durch zwei Dimethyldithiocarbamatliganden substituiert und zusätzlich insgesamt vier Phosphanliganden abgespalten werden. Im GCMS der Mutterlauge konnte weder der Thioether $\left(\mathrm{Br}-\mathrm{C}_{6} \mathrm{H}_{4}\right)_{2} \mathrm{~S}$ noch das Disulfid $\left(\mathrm{Br}-\mathrm{C}_{6} \mathrm{H}_{4}-\mathrm{S}\right)_{2}$ detektiert werden, was die Vermutung nahe legt, dass das $\left(\mathrm{Me}_{2} \mathrm{~N}-\mathrm{CS}_{2}\right)_{2}$ durch ein gebildetes Cuprat oder nicht abreagiertes ${ }^{i} \operatorname{PrMgCl}$ gespalten wird.

\section{Kristallstrukturen von $\mathbf{1 - 5}$}

Die Molekülstrukturen der Verbindungen wurden 1-5 mittels Einkristall-Röntgenstrukturanalyse ermittelt. Kristall- und Verfeinerungsdetails sind in Tabelle 1 zusammengefasst.

Verbindung 1 kristallisiert in der triklinen Raumgruppe $P \overline{1}$ mit einer Formeleinheit pro Elementarzelle. Weiterhin findet man in der Elementarzelle ein nicht koordiniertes DME-Molekül. Das Inversionszentrum befindet sich in der Mitte des aus Cu1, S1 und deren Symmetrieäquivalenten gebildeten Rings. Die Molekülstruktur von 1 ist in Abbildung 1 dargestellt. In der leiterartigen Struktur von 1 sind drei viergliedrige $\left[\mathrm{Cu}_{2} \mathrm{~S}_{2}\right]$-Ringe über gemeinsame Cu-S-Kanten verknüpft. Der zentrale viergliedrige Ring (Cu1, S1 und deren Symmetrieäquivalente) ist dabei planar, während die beiden Äußeren (Cu1, $\mathrm{Cu} 2, \mathrm{~S} 1$ und S2) in einer gefalteten Konformation vorliegen. In der Literatur sind vergleichbare Verbindungen bereits bekannt, wie z. B. $\left[\mathrm{Cu}_{4}(\mathrm{SPh})_{4}\left(\mathrm{PPh}_{3}\right)_{4}\right]$ [16] oder $\left[\mathrm{Cu}_{4}(\mathrm{SC}(\mathrm{O}) \mathrm{Me})_{4}\left(\mathrm{PPh}_{3}\right)_{4}\right]$.[17] Cu1 und dessen Symmetrieäquivalent sind von je drei $\mathrm{S}-\mathrm{C}_{6} \mathrm{H}_{4}-\mathrm{Br}$ - und einem Phosphanliganden koordiniert und weisen eine verzerrt tetraedrische Koordinationssphäre auf, während $\mathrm{Cu} 2$ und dessen Symmetrieäquivalent verzerrt trigonal-planar von zwei S- $\mathrm{C}_{6} \mathrm{H}_{4}-\mathrm{Br}-\mathrm{und}$ einem Phosphanliganden umgeben sind. Zwei der Thiolatoliganden koordinieren $\mu_{2}$ - und weitere zwei koordinieren $\mu_{3}$-verbrückend die Kupferatome. Die Kupfer-Schwefel-Abstände für die trigonal-planar koordinierten Kupferatome sind mit 228,9 bis 231,3 
pm erwartungsgemäß etwas kürzer, als die der tetraedrisch koordinierten Kupferatome mit Abständen zwischen 231,9 bis $251,9 \mathrm{pm}$

In vorangegangenen Arbeiten haben wir bereits über eine Serie von „Core-Shell“-Kupferchalkogenid-Cluster berichtet.[12,18] Verbindung 2 enthält 22 Kupferatome und kristallisiert in der monoklinen Raumgruppe $P 2_{1} / c$ mit zwei Formeleinheiten pro Elementarzelle. Weiterhin findet man pro Formeleinheit sechs nicht koordinierte Lösungsmittelmoleküle (DME). Der Kern von Verbindung 2 ist schalenartig aufgebaut, bestehend aus einem Kupferselenidkern der umgeben ist von einer Kupferthiolathülle. In Abbildung 2 ist die Molekülstruktur von 2 mit (rechts) und ohne Ligandenhülle (links), sowie die Chalkogenteilstruktur dargestellt (unten). Die Anordnung der Selenatome kann man als zwei kantenverknüpfte Tetraeder ansehen, während man die ganze Chalkogenteilstruktur als vier kantenverknüpfte Tetraeder beschreiben kann, die damit einen Ausschnitt aus einer hexagonal dichtesten Packung bilden. Auf zwei gegenüber liegenden Flächen der kantenverknüpften Tetraeder sind zwei Kupferatome (Cu1, Cu1') in einer verzerrt trigonal-planaren Koordination von drei Selenatomen umgeben, mit Cu-Se-Bindungsabständen zwischen 215,8 und 271,8 pm. Die beiden Kupferatome rücken dabei etwas aus der Dreiecksfläche hinaus in das Innere der Tetraeder. Zwei weitere gegenüber liegende Flächen werden von $\left[\mathrm{Cu}\left(\mathrm{PPh}_{3}\right)\right]$-Einheiten überkappt. $\mathrm{Cu} 2$ und $\mathrm{Cu} 2$ ' erhalten dadurch eine verzerrt tetraedrische Koordination von drei Selenatomen und einem Phosphoratom. Die Abstände zwischen den Selen- und den Kupferatomen reichen dabei von 250,4 bis 283,3 pm. Cu3, Cu5, Cu6 und deren Symmetrieäquivalente überkappen jeweils eine Se-SeKante und werden zusätzlich noch durch einen Thiolatoliganden koordiniert, was zur trigonal-planaren Koordination dieser Atome führt. Die Cu-Se-Abstände variieren dabei von 235,2 bis 246,7 pm. Cu4 überkappt ebenfalls eine solche Kante (CuSe: 248,9-271,1 pm) und ist zusätzlich noch von zwei weiteren Thiolatoliganden tetraedrisch koordiniert (Cu-S: 230,0240,8 pm). Auch Cu7, Cu11 und deren Symmetrieäquivalente sind tetraedrisch von drei Chalkogen- und einen Phosphoratom umgeben (Cu-Se: 273,1 pm; Cu-S: 231,4-265,3 pm). Die verbleibenden Kupferatome liegen alle in einer verzerrt trigonal-planaren Koordination vor und sind entweder von einem Selen-, einem Schwefel- und einem Phosphoratom koordiniert (Cu9 und Symmetrieäquivalent; Cu-Se: 244,6 pm; Cu-S: 230,4 pm) oder von zwei Schwefel- und einem Selenatom umgeben (Cu8, Cu10 und Symmetrieäquivalente; Cu-Se: 242,8-248,1 pm; Cu-S: 227,6-237,1 pm). Die KupferChalkogen-Abstände sind alle vergleichbar mit denen von ähnlichen Verbindungen, die aus der Literatur bereits bekannt sind.[18] Die Koordinationsmoden der Chalkogenatome in 2 variieren von $\mu_{6^{-}}$-verbrückend für alle Selenatome bis $\mu_{4^{-}}, \mu_{3^{-}}$ und $\mu_{2}$-verbrückend für die Schwefelatome der Thiolatoliganden.

Der Kern von Verbindung 3 ist ebenso wie $\mathbf{2}$ schalenartig aufgebaut, bestehend aus einem Kupferselenidkern der umgeben ist von einer Kupferthiolathülle. Verbindung 3 enthält 28 Kupferatome und kristallisiert in der monoklinen Raumgruppe $C c$ mit acht Formeleinheiten pro Elementarzelle und zwei unabhängigen Molekülen in der asymmetrischen Einheit. Beide Moleküle haben eine sehr ähnliche Molekülstruktur, sind allerdings aufgrund kleiner Unterschiede besonders in der Stellung 
der Phenylringe in der organischen Clusterhülle nicht deckungsgleich. Jedes der beiden Clustermoleküle ist zwar annähernd $\mathrm{C}_{2}$-symmetrisch, wobei allerdings auch diese Symmetrie nicht strikt erfüllt ist. Eine Verfeinerung in der zentrosymmetrischen Raumgruppe $C 2 / c$ ist daher nicht möglich. Dieser Sachverhalt wird auch durch Anwendung des Programmpaketes PlATON [14, 15] bestätigt, mit dessen Hilfe keine Hinweise für das Vorliegen einer höheren Symmetrie gefunden werden können. Die Qualität der Kristalle, die von 3 erhalten wurden, war durchwegs relativ gering, so dass trotz der röntgenographischen Untersuchung mehrerer Kristalle aus verschiedenen Syntheseansätzen nur Datensätze geringer Güte erhalten werden konnten (siehe Gütefaktoren in Tabelle 1). Dieses Problem ergibt sich allerdings häufig bei der strukturellen Untersuchung von großen Clustermolekülen, besonders dadurch bedingt, dass die entsprechend großen Zwischenräume zwischen den einzelnen annähernd kugelförmigen Clustern durch meist stark fehlgeordnete Lösungsmittelmoleküle ausgefüllt werden. In Abbildung 3 ist die Molekülstruktur eines der Moleküle von 3 mit (unten) und ohne Ligandenhülle (oben) dargestellt. Da sich die beiden kristallographisch unabhängigen Clustermoleküle in ihren Bindungsparametern nur unwesentlich unterscheiden, wird auf ein Darstellung und Diskussion des zweiten Moleküls verzichtet. Die Struktur der zu 3 ähnlichen Verbindung um $\left[\mathrm{Cu}_{28} \mathrm{Se}_{6}(\mathrm{SPh})_{16}\left(\mathrm{PPh}_{3}\right)_{8}\right]$. wurde bereits in einer früheren Arbeit vorgestellt.[18] Im Gegensatz zu diesem Cluster wurden die verbrückenden SPh-Liganden in 3 durch $p$-S-C $\mathrm{C}_{6} \mathrm{H}_{4}-\mathrm{Br}-\mathrm{Liganden}$ ersetzt. Bezogen auf die Struktur des Clusterkerns sind beide Verbindungen sehr ähnlich aufgebaut. Die Selensubstruktur kann als Anordnung von zwei kantenverknüpften Tetraedern beschrieben werden. Die Thiolathülle bestehend aus 16 Schwefelatomen umgibt den Selenkern vollständig und bildet dabei einen Polyeder, der aus Dreiecks- und Sechsecksflächen besteht. Die Kupferatome liegen in verzerrt linearer, verzerrt trigonal-planarer oder in verzerrt tetraedrischer Koordination vor. Die Kupfer-Chalkogen-Bindungsabstände liegen dabei je nach Koordination im erwarteten Bereich (Cu-Se: 226,7275,1 pm; Cu-S: 214,2-277,6 pm).

Verbindung 4 enthält 47 Kupferatome und kristallisiert in der orthorhombischen Raumgruppe Pbcn mit vier Formeleinheiten pro Elementarzelle. Eine zweizählige Symmetrieachse verläuft durch Cu4 und S11 parallel zur kristallographischen b-Achse. Der Kern von Verbindung $\mathbf{4}$ ist ebenfalls schalenartig aufgebaut und besteht wie bei $\mathbf{2}$ und $\mathbf{3}$ aus einem Kupferselenidkern, der umgeben ist von einer Kupferthiolathülle. In Abbildung 4 ist links der Kern von Verbindung 4, rechts der Cluster mit Ligandenhülle und unten die Chalkogenteilstruktur dargestellt. Die Anordnung von sechs der zehn Selenatome im Clusterkern kann man als drei flächenverknüpfte Tetraeder beschreiben (Se1, Se3, Se4 und Symmetrieäquivalente). Insgesamt ist die Chalkogenteilstruktur als Anordnung von flächen- und kantenverknüpften Tetraedern aufgebaut. Cu7, Cu16 und deren Symmetrieäquivalente sind verzerrt linear von jeweils zwei Selenatomen koordiniert, mit erwartungsgemäß kurzen Cu-Se-Abständen zwischen 233,4 und 241,8 pm. Dagegen sind Cu12, Cu13, Cu14 und deren Symmetrieäquivalente verzerrt trigonal-planar von drei Selenatomen umgeben (Cu-Se: 234,3-294,8 pm). Der innere $\mathrm{Cu}_{10} \mathrm{Se}_{10}-\mathrm{Kern}_{1}$ ist umgeben von einer Kupfer-Schwefel-Hülle, in der sich neben Selen auch noch Schwefel-, Sauerstoff und Phosphoratome in der Koordinationssphäre der Kupferatome befinden. So sind Cu6, Cu8, Cu18, Cu22 und deren Symmetrieäquivalente verzerrt 
trigonal-planar von zwei Schwefel- und einem Selenatom umgeben (Cu-Se: 236,6-259,9 pm; Cu-S: 220,2-232,6 pm). Cu4, $\mathrm{Cu} 9, \mathrm{Cu} 10, \mathrm{Cu} 11, \mathrm{Cu} 20$ und deren Symmetrieäquivalente sind verzerrt tetraedrisch von Schwefel- und Selenatomen koordiniert (Cu-Se: 249,2-282,1 pm; Cu-S: 226,8-255,5 pm). Die an Phosphanmoleküle gebundenen Kupferatome sind entweder verzerrt trigonal-planar von zwei Chalkogenatomen und einem Phosphoratom (Cu5, Cu23 und Symmetrieäquivalente; Cu-Se: 244,7 pm; Cu-S: 226,8-228,4 pm) oder verzerrt tetraedrisch von einem Selen-, einem Phosphor- und zwei Schwefelatomen (Cu3, Cu24 und Symmetrieäquivalente; Cu-Se: 253,2-270,5 pm; Cu-S: 238,4-242,4 pm) umgeben. Die verbleibenden 12 Kupferatome sind an die Sauerstoffatome der Acetatliganden gebunden und überkappen entweder eine Vierecksfläche (Cu15, Cu17, Cu19, Cu21 und Symmetrieäquivalente) oder jeweils zwei Dreiecksflächen des Chalkogenteilgerüsts (Cu1, $\mathrm{Cu} 2$ und Symmetrieäquivalente). $\mathrm{Cu} 1, \quad \mathrm{Cu} 2$ und deren Symmetrieäquivalente liegen dabei in einer verzerrt tetraedrischen Koordination vor, während die restlichen Kupferatome verzerrt trigonal-planar von zwei Chalkogenatomen und einem Sauerstoffatom des Acetatliganden umgeben sind $(\mathrm{Cu}-\mathrm{Se}$ : 242,1-277,8 pm; Cu-S: 222,5-257,1 pm). ${ }^{1}$ Die Koordinationsmoden der Selenatome in Verbindung 4 reichen von $\mu_{5^{-}}$ verbrückend (Se5, Se5'), über $\mu_{6}$-verbrückend (Se1, Se2, Se3 und Symmetrieäquivalente) bis hin zu $\mu_{8}$-verbrückend (Se4, Se4'). Die Schwefelatome der S- $\mathrm{C}_{6} \mathrm{H}_{4}-\mathrm{Br}-$ Gruppen koordinieren entweder $\mu_{3}$-verbrückend an die Kupferatome (S1-S5, S7, S9 und Symmetrieäquivalente) oder $\mu_{4}$-verbrückend (S6, S8, S10, S11 und Symmetrieäquivalente). Die an S11 gebundene $\mathrm{C}_{6} \mathrm{H}_{4}$-Br-Gruppe ist in Übereinstimmung mit der zweizähligen Symmetrieachse, die durch S11 und Cu4 verläuft, über zwei Positionen fehlgeordnet.

Verbindung 5 (Abb. 5) kristallisiert in der triklinen Raumgruppe $P \overline{1}$ mit einer Formeleinheit pro Elementarzelle. Das Schweratomgerüst der inversionssymmetrischen Verbindung besteht aus acht Kupferatomen und zehn Schwefelatomen, die sich zu einem Käfig aus viergliedrigen und sechsgliedrigen Ringen anordnen. Alle acht Kupferatome sind von jeweils drei Schwefelatomen der Thiolato- und Dithiocarbamatliganden umgeben. Vier der Kupferatome sind zusätzlich noch von einem Triphenylphophanliganden koordiniert, so dass diese in verzerrt tetraedrischer Koordination und die restlichen Kupferatome in verzerrt trigonal-planarer Koordination vorliegen. Alle Kupfer-Schwefel-Abstände der trigonal-planar koordinierten Kupferatome sind mit 224,0 bis 232,0 pm erwartungsgemäß etwas kürzer, als die der tetraedrisch koordinierten Kupferatome mit Abständen zwischen 230,5 und 253,5 pm. Die Schwefelatome der $\mathrm{S}_{-} \mathrm{C}_{6} \mathrm{H}_{4}-\mathrm{Br}$-Gruppen koordinieren entweder $\mu_{2}{ }^{-}$(S2, S2') oder $\mu_{3}$-verbrückend (S1, S1', S3 und $\mathrm{S} 3^{\prime}$ ) an die Kupferatome, während die Schwefelatome der Dithiocarbamatliganden alle $\mu_{2}$-verbrückend koordinieren.

\section{Experimenteller Teil}

\footnotetext{
${ }^{1}$ Aufgrund der hohen Auslenkungsparameter wurden Cu1, Cu2, Cu11 isotrop auf Splitlagen verfeinert.
} 
Alle Reaktionen erfolgten unter Ausschluss von Luft und Feuchtigkeit mit $\mathrm{N}_{2}$ als Inertgas. DME, THF und Diethylether wurden über Natrium mit Benzophenon destilliert. $n$-Hexan wurde über $\mathrm{LiAlH}_{4}$ getrocknet und anschließend destilliert. CuOAc [19] und $\mathrm{Se}\left(\mathrm{SiMe}_{3}\right)_{2}$ [20] wurden nach den Standardmethoden hergestellt. Tetramethylthiuramdisulfid, $\mathrm{PPh}_{3}$, MeLiLsg. (1,6 M in THF), ${ }^{i}$ PrMgCl-Lsg. (2,0 M in THF), Triethylamin, Chlortrimethylsilan und $p$-Br- $\mathrm{C}_{6} \mathrm{H}_{4}-\mathrm{SH}(\mathrm{Reinheit}$ ca. 95\%) wurden von kommerziellen Quellen bezogen. IR-Spektren wurden als KBr-Verreibung mit einem FT-IR-Spektrometer Spectrum GX von Perkin Elmer, die NMR-Spektren mit einem Spektrometer Av400 der Firma Bruker aufgenommen. Die Datensammlung für die Einkristall-Röntgenstrukturanalyse erfolgte mit einem STOE IPDS II Diffraktometer unter Verwendung von Mo-K $\mathrm{K}_{\alpha}$-Strahlung $(\lambda=0,71073 \AA)$. Strukturlösung und Verfeinerung gegen $F^{2}$ wurden mit dem Programmpaket shelxs/shelxl durchgeführt.[21]

Darstellung von $\boldsymbol{p}$-Br- $\mathbf{C}_{6} \mathbf{H}_{4}-\mathrm{SSiMe}_{3}$ : In einem $250 \mathrm{~mL}$ Zweihalskolben mit Hahn werden $24 \mathrm{~g}(127 \mathrm{mmol}) p$-Br- $\mathrm{C}_{6} \mathrm{H}_{4}-\mathrm{SH}$ in $60 \mathrm{~mL}$ Diethylether gelöst. Bei Raumtemperatur wird dazu eine Lösung von 12,8 mL (127 mmol) Triethylamin und 16 $\mathrm{mL}(127 \mathrm{mmol})$ Chlortrimethylsilan in $40 \mathrm{~mL}$ Diethylether getropft, wobei sich ein farbloser Niederschlag bildet. Das Reaktionsgemisch wird 2 Stunden zum Sieden erhitzt, nach dem Abkühlen filtriert, und der Niederschlag mehrmals mit Diethylether gewaschen. Die flüchtigen Bestandteile des Filtrats und der Waschlösung werden im Vakuum entfernt und das Produkt als farblose Flüssigkeit durch fraktionierte Destillation im Vakuum gereinigt (Sdp. $66^{\circ} \mathrm{C} / 10^{-1}$ Torr); Ausbeute: 30,5 g (117 mmol, 92\%).

${ }^{1} \mathrm{H}-\mathrm{NMR}\left(400 \mathrm{MHz}, \mathrm{C}_{6} \mathrm{D}_{6}, 25{ }^{\circ} \mathrm{C}\right): \delta_{\mathrm{H}}=0,09\left(\mathrm{~s}, 9 \mathrm{H},-\mathrm{SSi}\left(\mathrm{CH}_{3}\right)_{3}\right), 7,08\left(\mathrm{~s}, 4 \mathrm{H}\right.$, Aryl-H) ppm. ${ }^{13} \mathrm{C}\left\{{ }^{1} \mathrm{H}\right\}-\mathrm{NMR}(100 \mathrm{MHz}$, $\left.\mathrm{C}_{6} \mathrm{D}_{6}, 25{ }^{\circ} \mathrm{C}\right): \delta_{\mathrm{C}}=1,5\left(\mathrm{~s}, \mathrm{SSiCH}_{3}\right), 122,3(\mathrm{~s}, \mathrm{Br}-\mathrm{C}), 132,1(\mathrm{~s}, \mathrm{~S}-\mathrm{C}), 132,9(\mathrm{~s}, o-\mathrm{Br}-\mathrm{C}), 137,8(\mathrm{~s}, o-\mathrm{S}-\mathrm{C}) \mathrm{ppm} .{ }^{29} \mathrm{Si}-\mathrm{NMR}(79$ $\left.\mathrm{MHz}, \mathrm{C}_{6} \mathrm{D}_{6}, 25^{\circ} \mathrm{C}\right): \delta_{\mathrm{Si}}=17,5 \mathrm{ppm}\left(\mathrm{dz},{ }^{2} J_{\mathrm{SiH}}=6,8 \mathrm{~Hz}\right)$.

Darstellung von $\left[\mathbf{C u}_{4}\left(\boldsymbol{p}-\mathbf{S}-\mathbf{C}_{6} \mathbf{H}_{4}-\mathbf{B r}\right)_{4}\left(\mathbf{P P h}_{3}\right)_{4}\right]$ (1): $\mathrm{Zu}$ einer Lösung von $123 \mathrm{mg}(1,00 \mathrm{mmol}) \mathrm{CuOAc}$ und $262 \mathrm{mg}(1,00$ mmol) $\mathrm{PPh}_{3}$ in $10 \mathrm{~mL}$ DME werden 0,260 mL (1,00 mmol) $\mathrm{Me}_{3} \mathrm{SiS}-\mathrm{C}_{6} \mathrm{H}_{4}-\mathrm{Br}$ gegeben. Die entstandene gelbe Lösung lässt man für weitere 16 Stunden rühren und überschichtet anschließend mit $n$-Hexan. Nach wenigen Tagen erhält man farblose Kristalle der Zusammensetzung 1·DME. Ausbeute: $176 \mathrm{mg}(0,16 \mathrm{mmol}, 66 \%$ bezogen auf CuOAc). Elem.-Analyse: ber. C, 56,$09 ; \mathrm{H}, 3,73 ;$ S 6,24. gef. C, 56,21; H 3,92\%; S 6,15.

IR $\left(\mathrm{KBr} / \mathrm{cm}^{-1}\right): 3056(\mathrm{~m}), 2737(\mathrm{w}), 1955(\mathrm{w}), 1884(\mathrm{w}), 1811(\mathrm{w}), 1770(\mathrm{w}), 1661(\mathrm{w}), 1585(\mathrm{w}), 1572(\mathrm{w}), 1557(\mathrm{w}), 1479$ (m), 1467 (s), 1434 (s), 1380 (m), 1327 (w), 1308 (w), 1285 (w), 1262 (w), $1181(w), 1157$ (w), 1082 (s), 1068 (m), 1026 (w), 1006 (s), 917 (w), 847 (w), 807 (s), 741 (s), 693 (s), 618 (w), $521(\mathrm{~s}), 483(\mathrm{~s}), 442(\mathrm{w})$.

Darstellung von $\left[\mathbf{C u}_{22} \mathbf{S e}_{6}\left(\boldsymbol{p}-\mathbf{S}-\mathbf{C}_{6} \mathbf{H}_{4}-\mathbf{B r}\right)_{10}\left(\mathbf{P P h}_{3}\right)_{8}\right]$ (2): Zu einer Lösung von $318 \mathrm{mg}(2,6 \mathrm{mmol}) \mathrm{CuOAc}$ und $570 \mathrm{mg}(2,16$ mmol) $\mathrm{PPh}_{3}$ in $50 \mathrm{~mL}$ DME werden $0,36 \mathrm{~mL}(1,39 \mathrm{mmol})$ p-Me $\mathrm{Me}_{3} \mathrm{SiS}-\mathrm{C}_{6} \mathrm{H}_{4}-\mathrm{Br}$ und $0.10 \mathrm{~mL}(0,43 \mathrm{mmol}) \mathrm{Se}(\mathrm{SiMe})_{2}$ gegeben. Die entstandene rote Lösung lässt man bei Raumtemperatur stehen. Nach wenigen Tagen erhält man rote 
stäbchenförmige Kristalle von 2.6DME. Ausbeute: $453 \mathrm{mg}\left(7,1 \cdot 10^{-2} \mathrm{mmol}, 60 \%\right.$ bezogen auf CuOAc). Elem.-Analyse: ber. C 41,71; H 3,09; S 5,46. gef. C 41,96; H 3,35; S 5,34\%.

IR $\left(\mathrm{KBr} / \mathrm{cm}^{-1}\right)$ : $3051(\mathrm{w}), 3003(\mathrm{w}), 2963(\mathrm{w}), 2927(\mathrm{w}), 2292(\mathrm{w}), 2226(\mathrm{w}), 1956(\mathrm{w}), 1884(\mathrm{w}), 1626(\mathrm{w}), 1586(\mathrm{w}), 1479$ (w), $1466(\mathrm{~s}), 1434(\mathrm{~s}), 1381(\mathrm{w}), 1309(\mathrm{w}), 1262(\mathrm{w}), 1183(\mathrm{w}), 1158(\mathrm{w}), 1095(\mathrm{~m}), 1081(\mathrm{~s}), 1027(\mathrm{w}), 1006(\mathrm{~s}), 808(\mathrm{~m})$, $743(\mathrm{~m}), 693(\mathrm{~s}), 618(\mathrm{w}), 521(\mathrm{~s}), 488(\mathrm{~m})$.

Darstellung von $\left[\mathbf{C u}_{28} \mathbf{S e}_{6}\left(\boldsymbol{p}-\mathbf{S}-\mathbf{C}_{6} \mathbf{H}_{4}-\mathbf{B r}\right)_{16}\left(\mathbf{P P h}_{3}\right)_{8}\right]$ (3): Zu einer Suspension von $184 \mathrm{mg}(1,50 \mathrm{mmol}) \mathrm{CuOAc}$ und $131 \mathrm{mg}$ (0,50 mmol) $\mathrm{PPh}_{3}$ in $25 \mathrm{~mL}$ DME werden 0,195 mL (0,75 mmol) $\mathrm{Me}_{3} \mathrm{SiS}-\mathrm{C}_{6} \mathrm{H}_{4}-\mathrm{Br}$ und 0,065 mL $(0,26 \mathrm{mmol}) \mathrm{Se}(\mathrm{SiMe})_{2}$ gegeben. Die entstandene rote Lösung lässt man bei Raumtemperatur stehen. Nach wenigen Tagen erhält man rote Kristalle von 3. Ausbeute: $35 \mathrm{mg}\left(4,8 \cdot 10^{-3} \mathrm{mmol}, 9 \%\right.$ bezogen auf CuOAc). Elem.-Analyse: ber. C 39,16; H 2,52; S 6.97. gef. C 38,$56 ; \mathrm{H} 2,68 ; \mathrm{S} 7.05 \%$.

IR $\left(\mathrm{KBr} / \mathrm{cm}^{-1}\right): 3052(\mathrm{~m}), 3003(\mathrm{w}), 2961(\mathrm{w}), 2925(\mathrm{w}), 1957(\mathrm{w}), 1884(\mathrm{w}), 1812(\mathrm{w}), 1585(\mathrm{w}), 560(\mathrm{~m}), 1479(\mathrm{~m}), 1467$ (s), 1435 (s), $1381(\mathrm{~m}), 1328(\mathrm{w}), 1309$ (w), $1288(w), 1261(\mathrm{~m}), 1181(\mathrm{w}), 1157$ (w), $1095(\mathrm{~s}), 1081$ (s), $1069(\mathrm{~s}), 1027$ (m), $1006(\mathrm{~s}), 873(\mathrm{w}), 846(\mathrm{w}), 808(\mathrm{~s}), 743(\mathrm{~s}), 722(\mathrm{w}), 693(\mathrm{~s}), 665(\mathrm{w}), 618(\mathrm{w}), 522(\mathrm{~s}), 485(\mathrm{~s})$.

Darstellung von $\left[\mathbf{C u}_{47} \mathbf{S e}_{10}\left(\boldsymbol{p}-\mathbf{S}-\mathbf{C}_{6} \mathbf{H}_{4}-\mathbf{B r}\right)_{21}(\mathbf{O A c})_{6}\left(\mathbf{P P h}_{3}\right)_{8}\right](4)$ : $\mathrm{Zu}$ einer Suspension von $184 \mathrm{mg}(1,50 \mathrm{mmol}) \mathrm{CuOAc}$ und $98 \mathrm{mg}(0,38 \mathrm{mmol}) \mathrm{PPh}_{3}$ in $25 \mathrm{~mL}$ DME werden $0,195 \mathrm{~mL}(0,75 \mathrm{mmol}) \mathrm{Me}_{3} \mathrm{SiS}_{-} \mathrm{C}_{6} \mathrm{H}_{4}-\mathrm{Br}$ und $0,030 \mathrm{~mL}(0,12 \mathrm{mmol})$ $\mathrm{Se}\left(\mathrm{SiMe}_{3}\right)_{2}$ gegeben. Die entstandene rote Suspension lässt man für weitere 16 Stunden rühren und filtriert diese anschließend. Nach wenigen Tagen erhält man wenige rote Kristalle von 4. Ausbeute: $13 \mathrm{mg}\left(1,3 \cdot 10^{-3} \mathrm{mmol}, 4 \%\right.$ bezogen auf CuOAc). Elem.-Analyse: ber. C 33,28; H 2,20. gef. C 33,39; H 2,25\%.

IR $\left(\mathrm{KBr} / \mathrm{cm}^{-1}\right): 3423(\mathrm{w}), 3052(\mathrm{w}), 2963(\mathrm{w}), 2369(\mathrm{w}), 2346(\mathrm{w}), 1885(\mathrm{w}), 1625(\mathrm{~m}), 1560(\mathrm{~m}), 1468(\mathrm{~s}), 1435(\mathrm{~m}), 1381$ (m), $1289(\mathrm{w}), 1261(\mathrm{~m}), 1177(\mathrm{w}), 1095(\mathrm{~m}), 1081(\mathrm{~s}), 1069(\mathrm{~m}), 1026(\mathrm{~m}), 1006(\mathrm{~s}), 806(\mathrm{~s}), 743(\mathrm{~m}), 722(\mathrm{w}), 693(\mathrm{~m})$, $623(\mathrm{w}), 541(\mathrm{w}), 523(\mathrm{~m}), 484(\mathrm{~m})$.

Darstellung von $\left[\mathbf{C u}_{8}\left(\boldsymbol{p}-\mathbf{S}-\mathbf{C}_{6} \mathbf{H}_{4}-\mathbf{B r}\right)_{6}\left(\mathbf{S}_{2} \mathbf{C}-\mathbf{N M e}_{2}\right)_{2}\left(\mathbf{P P h}_{3}\right)_{4}\right](\mathbf{5})$ : $\mathrm{Zu}$ einer Lösung von $50 \mathrm{mg}(0,025 \mathrm{mmol})\left[\mathrm{Cu}_{4}\left(p-\mathrm{S}-\mathrm{C}_{6} \mathrm{H}_{4}-\right.\right.$ $\left.\mathrm{Br})_{4}\left(\mathrm{PPh}_{3}\right)_{4}\right](\mathbf{1})$ in $10 \mathrm{~mL}$ DME gibt man bei $-70{ }^{\circ} \mathrm{C} 0,025 \mathrm{~mL}(0,05 \mathrm{mmol})$ einer zweimolaren Lösung von ${ }^{i} \mathrm{PrMgCl}$ in $\mathrm{THF}$ und lässt innerhalb von vier Stunden auf $10{ }^{\circ} \mathrm{C}$ erwärmen. Zu der entstandenen bräunlichen Suspension gibt man 12 mg (0,050 mmol) $\mathrm{Me}_{2} \mathrm{~N}-\mathrm{C}(\mathrm{S})-\mathrm{S}-\mathrm{S}-\mathrm{C}(\mathrm{S})-\mathrm{NMe}_{2}$. Die entstandene gelbe Lösung überschichtet man anschließend mit ${ }^{n} \mathrm{Hexan} . \mathrm{Nach}$ wenigen Tagen erhält man gelbe Kristalle. Ausbeute: $26 \mathrm{mg}\left(8,9 \cdot 10^{-3} \mathrm{mmol}, 73 \%\right.$ bezogen auf 1). Elem.-Analyse: ber. C 46,79; H 3,31; N 0,96; S 10,96. gef. C 46,98; H 3,25; N 1,06; S $11.08 \%$.

IR $\left(\mathrm{KBr} / \mathrm{cm}^{-1}\right): 3048(\mathrm{~m}), 2921(\mathrm{~m}), 1884(\mathrm{w}), 1633(\mathrm{w}), 1585$ (w), $1571(\mathrm{w}), 1497$ (s), $1480(\mathrm{~s}), 1465$ (s), 1434 (s), 1399 (w), $1372(\mathrm{~s}), 1244(\mathrm{~m}), 1182(\mathrm{w}), 1139(\mathrm{~m}), 1094(\mathrm{~m}), 1082(\mathrm{~s}), 1026(\mathrm{w}), 1006(\mathrm{~m}), 962(\mathrm{~s}), 874(\mathrm{w}), 810(\mathrm{~m}), 743$ (s), 693 (s), $563(\mathrm{w}), 520(\mathrm{~s}), 505(\mathrm{~m}), 494(\mathrm{~m}), 441(\mathrm{w})$. 


\section{Danksagung}

Diese Arbeit wurde von der Deutschen Forschungsgemeinschaft (Centrum für funktionelle Nanostrukturen, CFN) gefördert.

[1] C. Burda, X. Chen, R. Narayanan, M. A. El Slayed, Chem.Rev. 2005, 105, 1025-1102.

[2] C. Krüger, A. Seema, A. Greiner, J. Am. Chem. Soc. 2008, 130, 2710-2711.

[3] A. Müller, E. Beckmann, H. Bögge, M. Schmidtmann, A. Dress, Angew. Chem. 2002, 114, 1210-1215; Angew. Chem. Int. Ed. 2002, $41,1161-1167$.

[4] N. T. Tran, D. R. Powell, L. F. Dahl, Angew. Chem. 2000, 112, 4287-4291; Angew. Chem. Int. Ed. 2000, 39, 4121-4125.

[5] C. E. Anson, A. Eichhöfer, I. Issac, D. Fenske, O. Fuhr, P. Sevillano, C. Persau, D. Stalke, J. Zhang, Angew. Chem., 2008, 120, 1346-1351; Angew. Chem. Int. Ed., 2008, 47, 1326-1331.

[6] S. Dehnen, A. Eichhöfer, D. Fenske, Eur. J. Inorg. Chem. 2002, 279-317 und darin zitierte Arbeiten.

[7] S. Schneider, J. A. S. Roberts, M. R. Salata, T. J. Marks, Angew. Chem 2006, 118, 1765-1768; Angew. Chem. Int. Ed. 2006, 45, $1733-1736$.

[8] C. Femoni, M. C. Iapalucci, G. Longoni, C. Tiozzo, S. Zacchini, Angew. Chem 2008, 120, 6768-6771; Angew. Chem. Int. Ed. 2006, 47, 6666-6669.

[9] G. Schmid, N. Klein, L. Korste, U. Kreibig, D. Schoenauer, Polyhedron 1988, 7, 605-608.

[10] O. Fuhr, A. Meredith, D. Fenske, J. Chem. Soc., Dalton Trans. 2002, 4091-4094.

[11] S. Chitaz, D. Fenske, O. Fuhr, Angew. Chem. 8224-8228;-

[12] L. Fernández-Recio, D. Fenske, O. Fuhr, Z. Anorg. Allg. Chem. 2008, 634, 2553-2857.

[13] L. Fernandez-Recio, Dissertation 2006.

[14] A. L. Spek, J. Appl. Christallogr. 2003, 36, 7-13.

[15] J. P. van der Sluis, A. L. Spek, Acta Chrystallogr. Sect. A 1990, 46, 194-201.

[16] I. G: Dance, M. Scudder, L. Fitzpatrick, Inorg. Chem. 1985, 24, 2547-2550.

[17] T. C. Deivaraj, G. X. Lai, J. J. Vittal, Inorg. Chem. 2000, 39, 1028-1034.

[18] O. Fuhr, L. Fernandez-Recio, D. Fenske, Eur. J. Inorg. Chem. 2005, 2306-2314

[19] D. A. Edwards, R. Richards, J. Chem. Soc., Dalton Trans. 1973, 2463-2468.

[20] H. Schmidt, H. Ruf, Z. Anorg. Allg. Chem. 1963, 321, 270-273. 


\section{Page 11 of 15}

1

2

3

4

5

6

7

8

9

10

11

12

13

14

15

16

17

18

19

20

21

22

23

24

25

26

27

28

29

30

31

32

33

34

35

36

37

38

39

40

41

42

43

44

45

46

47

48

49

50

51

52

53

54

55

56

57

58

59

60

[21] (a) G. M. Sheldrick, SHELXL-97. Program for the Refinement of Crystal Structures; University of Goettingen: Germany, 1997; (b)

G. M. Sheldrick, SHELXS-97. Program for the Refinement of Crystal Structures; University of Goettingen: Germany, 1997

Received: ((will be filled in by the editorial staff)) Published online: ((will be filled in by the editorial staff)) 
Tabelle 1. Kristallographische Daten der Verbindung 1-5

\begin{tabular}{|c|c|c|c|c|c|}
\hline Verbindung & 1.DME & 2.6DME & $3^{[\mathrm{a}]}$ & $4^{[\mathrm{a}]}$ & $5^{[\mathrm{a}]}$ \\
\hline Zusammensetzung & $\mathrm{C}_{100} \mathrm{H}_{86} \mathrm{P}_{4} \mathrm{~S}_{4} \mathrm{Cu}_{4} \mathrm{Br}_{4} \mathrm{O}_{2}$ & $\mathrm{C}_{228} \mathrm{H}_{220} \mathrm{O}_{12} \mathrm{P}_{8} \mathrm{~S}_{10} \mathrm{Cu}_{22} \mathrm{Br}_{10} \mathrm{Se}_{6}$ & $\mathrm{C}_{240} \mathrm{H}_{184} \mathrm{P}_{8} \mathrm{~S}_{16} \mathrm{Cu}_{28} \mathrm{Br}_{16} \mathrm{Se}_{6}$ & $\mathrm{C}_{282} \mathrm{H}_{222} \mathrm{O}_{12} \mathrm{P}_{8} \mathrm{~S}_{21} \mathrm{Cu}_{47} \mathrm{Br}_{21} \mathrm{Se}_{10}$ & $\mathrm{C}_{114} \mathrm{H}_{96} \mathrm{~N}_{2} \mathrm{P}_{4} \mathrm{~S}_{10} \mathrm{Cu}_{8} \mathrm{Br}_{6}$ \\
\hline $\mathrm{M} / \mathrm{g} \mathrm{mol}^{-1}$ & 2145,61 & 6391,14 & 7360,03 & 10177,71 & 2936,19 \\
\hline Kristallsystem & triklin & monoklin & monoklin & orthorhombisch & triklin \\
\hline Raumgruppe & $P \overline{1}$ & $P 2_{1} / n$ & $C c$ & $P b c n$ & $P \overline{1}$ \\
\hline $\mathrm{Z}$ & 1 & 2 & 8 & 4 & 1 \\
\hline $\mathrm{T} / \mathrm{K}$ & 180 & 200 & 180 & 150 & 120 \\
\hline $\mathrm{a}, \mathrm{b}, \mathrm{c} / \mathrm{pm}$ & $1372,1(3) ; 1382,6(3) ; 1493,0(3)$ & $1836,5(4) ; 3827,3(8) ; 1902,0(4)$ & $4674,3(9) ; 4310,3(9) ; 3252,4(7)$ & $3781,7(8) ; 3320,8(7) ; 2875,7(6)$ & $1288,1(3) ; 1521,6(3) ; 1714,3(3)$ \\
\hline$\alpha, \beta, \gamma /^{\circ}$ & $102,03(3) ; 107,62(3) ; 113,87(3)$ & $90 ; 116,22(3) ; 90$ & $90 ; 109,24(3) ; 90$ & $90 ; 90 ; 90$ & $109,13(3) ; 93,68(3) ; 99,08(3)$ \\
\hline $\mathrm{V} / 10^{6} \mathrm{pm}^{3}$ & $2283,7(8)$ & 11993(4) & $61870(21)$ & $36115(13)$ & $3110,4(11)$ \\
\hline Dichte/g cm ${ }^{-3}$ & 1,560 & 1,770 & 1,580 & 1,872 & 1,562 \\
\hline$\mu / \mathrm{mm}^{-1}$ & 2,879 & 4,675 & 4,841 & 6,233 & 3,530 \\
\hline Gemessene Reflexe & 11212 & 58219 & 158854 & 81267 & 14507 \\
\hline Unabhängige Reflexe & $6253\left[\mathrm{R}_{\mathrm{int}}=0,02223\right]$ & $23527\left[\mathrm{R}_{\mathrm{int}}=0,0660\right]$ & $78749\left[\mathrm{R}_{\mathrm{int}}=0,0874\right]$ & $25702\left[\mathrm{R}_{\mathrm{int}}=0,1038\right]$ & $10204\left[\mathrm{R}_{\mathrm{int}}=0,0410\right]$ \\
\hline Unabh. Refl. mit I $>2 \sigma(\mathrm{I})$ & 5467 & 14605 & 63397 & 17767 & 6771 \\
\hline Parameter/Restrains & $684 / 0$ & $1483 / 0$ & $5381 / 325$ & $1792 / 0$ & $649 / 0$ \\
\hline Goof & 1.163 & 0.866 & 0,991 & 1,017 & 1,016 \\
\hline R-Werte $(\mathrm{I}>2 \sigma(\mathrm{I}))$ & $R 1=0,0290 ; w R 2=0,0778$ & $R 1=0,0485 ; w R 2=0,1125$ & $R 1=0,0933 ; w R 2=0,2426$ & $R 1=0,0649 ; w R 2=0,1641$ & $R 1=0,0646 ; w R 2=0,1641$ \\
\hline R-Werte (alle Daten) & $R 1=0,0360 ; w R 2=0,0801$ & $R 1=0,0806 ; w R 2=0,1249$ & $R 1=0,1089 ; w R 2=0,2587$ & $R 1=0,0916 ; w R 2=0,1773$ & $R 1=0,0946 ; w R 2=0,1784$ \\
\hline Restelektronendicht $/ \mathrm{e}^{-3}$ & $0,639 /-0,629$ & $1,181 /-1,111$ & $2,572 /-1,487$ & $3,965 /-2,083$ & $0,838 /-0,992$ \\
\hline Flack-Parameter & - & - & $0,261(9)$ & - & - \\
\hline CCDC-Nummer & 728096 & 728097 & 728098 & 728099 & 728100 \\
\hline
\end{tabular}

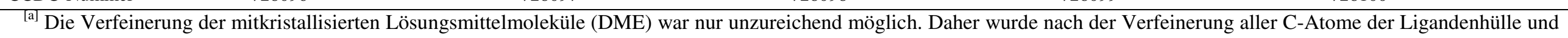

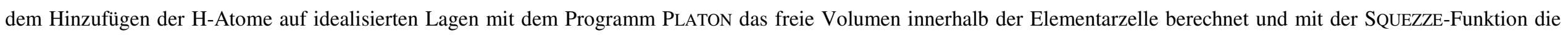

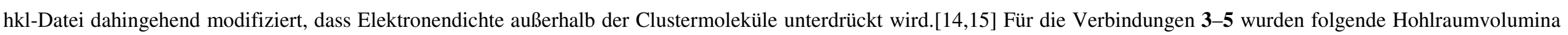

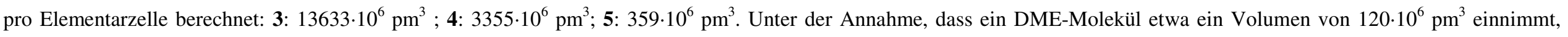

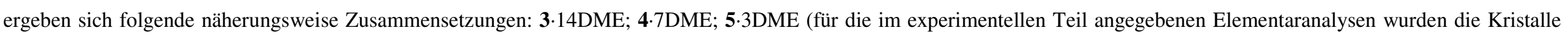

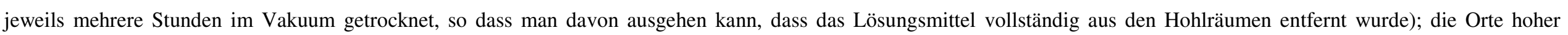

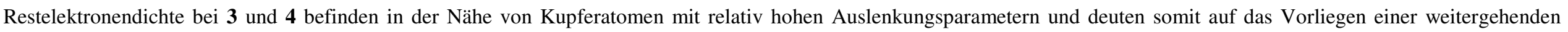
Fehlordnung hin. 
a CuOAc $\left[\mathrm{Cu}_{4}\left(\mathrm{~S}-\mathrm{C}_{6} \mathrm{H}_{4}-\mathrm{Br}\right)_{4}\left(\mathrm{PPh}_{3}\right)_{4}\right](\mathbf{1})$

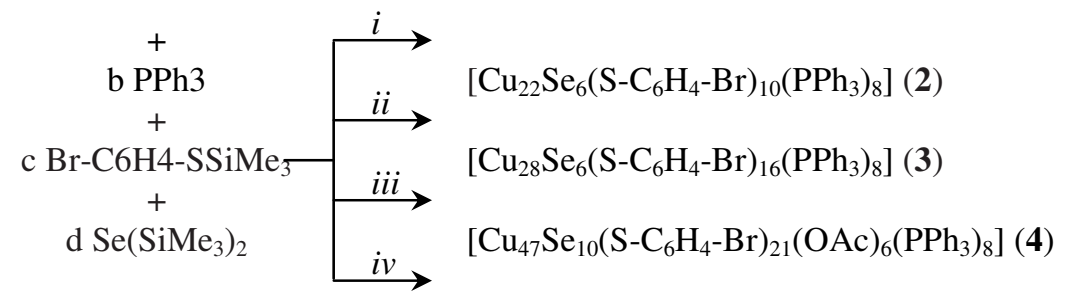

Schema 1. Reaktionen, die zu den Verbindungen 1-4 führen; eingesetzte molare Verhältnisse a:b:c:d i) 1:1:1:0 ii) 30:25:16:5 iii) 6:2:3:1 iv) $12: 3: 6: 1$.

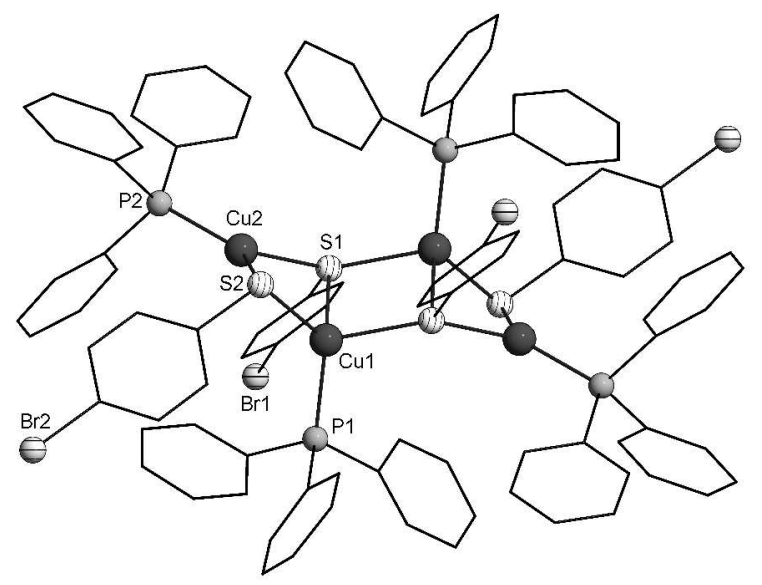

Abbildung 1. Molekülstruktur von $\left[\mathrm{Cu}_{4}\left(p-\mathrm{S}-\mathrm{C}_{6} \mathrm{H}_{4}-\mathrm{Br}\right)_{4}\left(\mathrm{PPh}_{3}\right)_{4}\right]$ (1) (H-Atome wurden aus Gründen der Übersicht weggelassen; nur die Atome der asymmetrischen Einheit sind benannt).

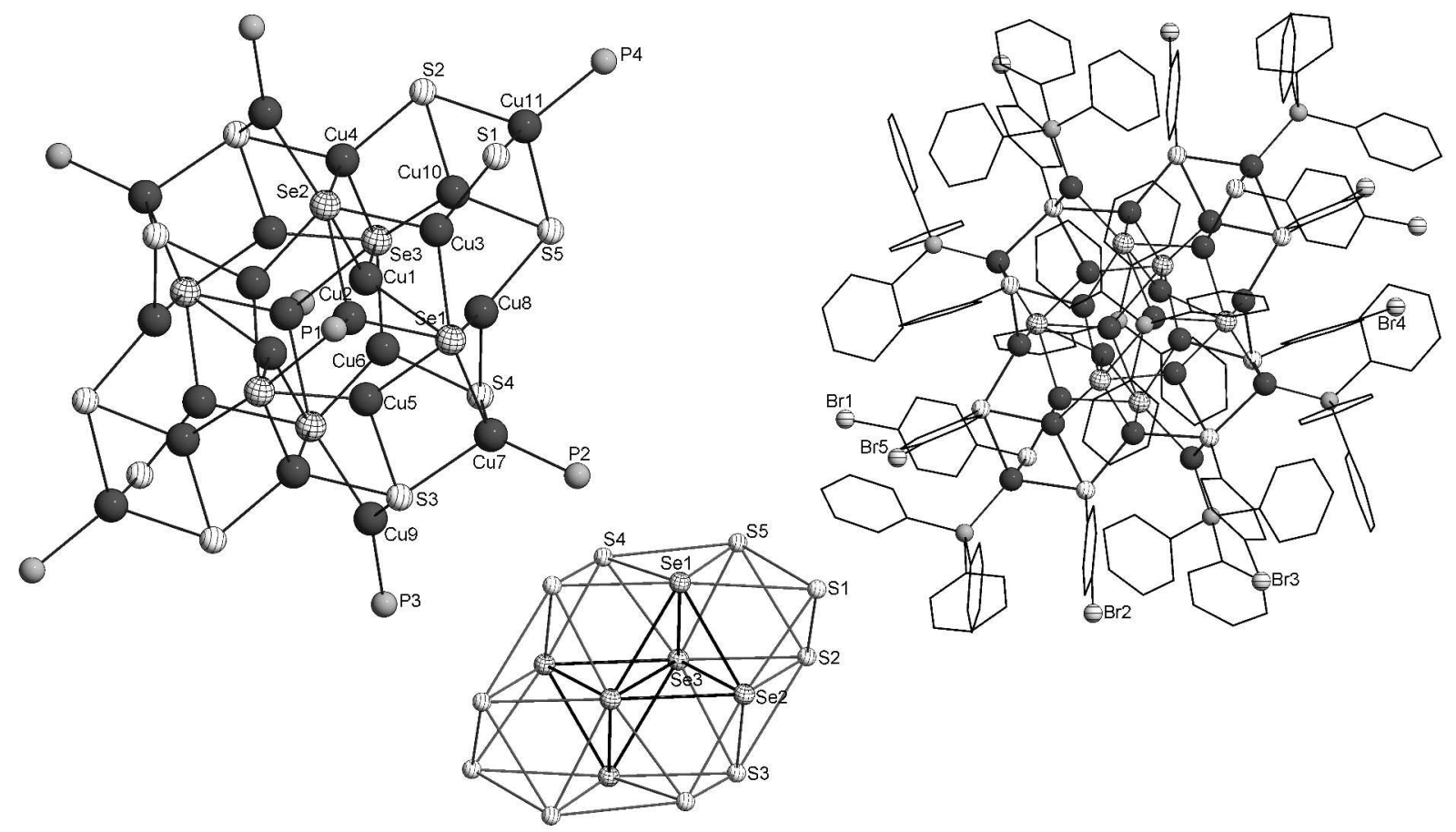

Abbildung 2. Die Molekülstruktur des Clusterkerns von $\left[\mathrm{Cu}_{22} \mathrm{Se}_{6}\left(p-\mathrm{S}-\mathrm{C}_{6} \mathrm{H}_{4}-\mathrm{Br}\right)_{10}\left(\mathrm{PPh}_{3}\right)_{8}\right]$ (2) (links), das Chalkogenteilgitter (unten) und der Cluster mit Ligandenhülle (rechts). (H-Atome wurden aus Gründen der Übersicht weggelassen; nur die Atome der asymmetrischen Einheit sind benannt). 

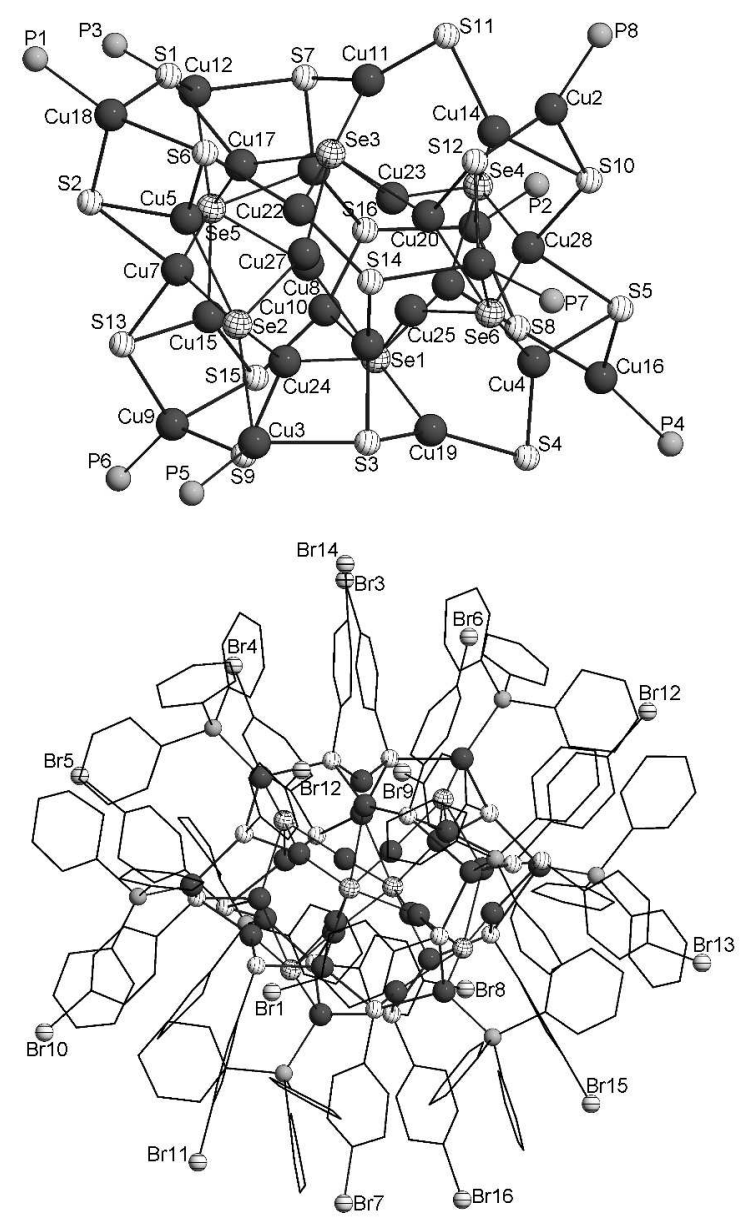

Abbildung 3. Die Molekülstruktur des Clusterkerns von $\left[\mathrm{Cu}_{28} \mathrm{Se}_{6}\left(p-\mathrm{S}-\mathrm{C}_{6} \mathrm{H}_{4}-\mathrm{Br}\right)_{16}\left(\mathrm{PPh}_{3}\right)_{8}\right]$ (3) (oben) und der Cluster mit Ligandenhülle (unten). (H-Atome wurden aus Gründen der Übersicht weggelassen.) 


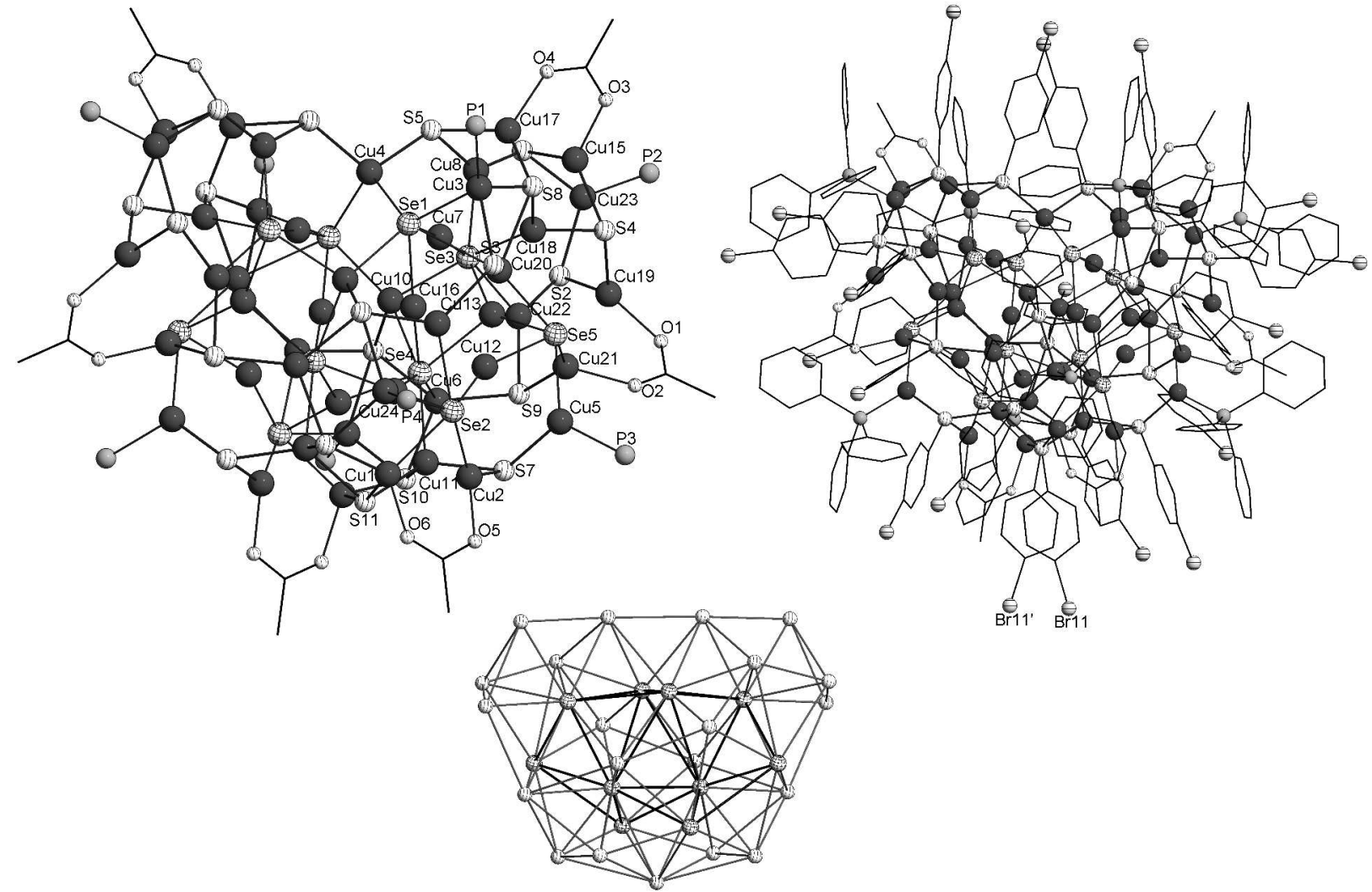

Abbildung 4. Die Molekülstruktur des Clusterkerns von $\left[\mathrm{Cu}_{47} \mathrm{Se}_{10}\left(p-\mathrm{S}-\mathrm{C}_{6} \mathrm{H}_{4}-\mathrm{Br}\right)_{21}(\mathrm{OAc})_{6}\left(\mathrm{PPh}_{3}\right)_{8}\right]$ (4) (links), das Chalkogenteilgitter (unten) und der Cluster mit Ligandenhülle (rechts). (H-Atome wurden aus Gründen der Übersicht weggelassen).

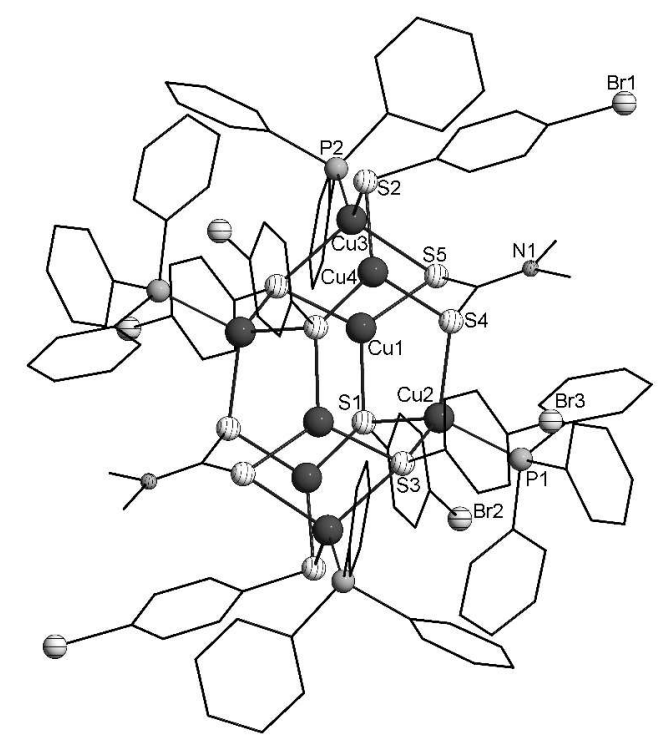

Abbildung 5. Molekülstruktur von $\left[\mathrm{Cu}_{8}\left(p-\mathrm{S}-\mathrm{C}_{6} \mathrm{H}_{4}-\mathrm{Br}\right)_{6}\left(\mathrm{~S}_{2} \mathrm{C}-\mathrm{NMe}_{2}\right)_{2}\left(\mathrm{PPh}_{3}\right)_{4}\right]$ (5). (H-Atome wurden aus Gründen der Übersicht weggelassen.) 